\title{
Deficiency of sphingomyelin synthase 1 but not sphingomyelin synthase 2 reduces bone formation due to impaired osteoblast differentiation
}

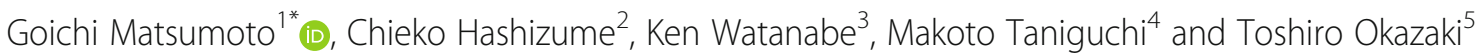

\begin{abstract}
Background: There are two isoforms of sphingomyelin synthase (SMS): SMS1 and SMS2. SMS1 is located in the Golgi apparatus only while SMS2 is located in both the plasma membrane and the Golgi apparatus. SMS1 and SMS2 act similarly to generate sphingomyelin (SM). We have undertaken the experiments reported here on SMS and osteoblast differentiation in order to better understand the role SMS plays in skeletal development.

Methods: We analyzed the phenotype of a conditional knockout mouse, which was generated by mating a Sp7 promoter-driven Cre-expressing mouse with an SMS1-floxed SMS2-deficient mouse (Sp7-Cre;SMS1/f; $S M S 2^{-1-}$ mouse).

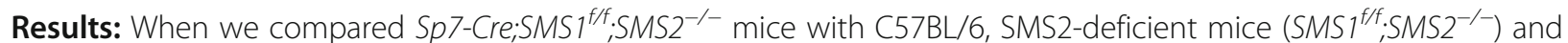
SP7-Cre positive control mice (Sp7-Cre, Sp7-Cre;SMS1 ${ }^{+/+} ; \mathrm{SMS2}^{+/-}$and Sp7-Cre;SMS1 ${ }^{+/+} ; \mathrm{SMS2}^{-/-}$), we found that

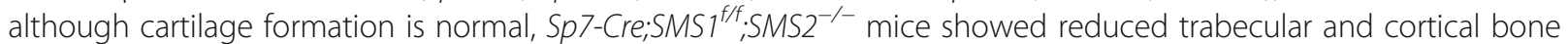
mass, had lower bone mineral density, and had a slower mineral apposition rate than control mice. Next, we have used a tamoxifen-inducible knockout system in vitro to show that SMS1 plays an important role in osteoblast differentiation. We cultured osteoblasts derived from ERT2-Cre;SMS1/f SMS2 ${ }^{-1-}$ mice. We observed impaired differentiation of these cells in response to Smad1/5/8 and p38 that were induced by bone morphogenic protein 2 (BMP2). However, Erk1/2 phosphorylation was unaffected by inactivation of SMS1.

Conclusions: These findings provide the first genetic evidence that SMS1 plays a role in bone development by regulating osteoblast development in cooperation with BMP2 signaling. Thus, SMS1 acts as an endogenous signaling component necessary for bone formation.
\end{abstract}

Keywords: Sphingomyelin synthase 1, Sphingomyelin synthase 2, SP7, Osteoblast differentiation, Bone morphogenic protein 2, Smad1/5/8, p38

\section{Introduction}

Skeletal homeostasis requires a precise balance between bone-forming osteoblasts and bone-resorbing osteoclasts. Osteoblasts differentiate and produce the bone matrix during skeletal development (Ducy et al. 2000). Osteoblast differentiation is regulated by various transcriptional factors, such as runt-related transcription factor-2 (Runx2) and Sp7 (osterix), which have been

\footnotetext{
* Correspondence: gmatsu@kanazawa-med.ac.jp

${ }^{1}$ Department of Oral and Maxillofacial Surgery, Kanazawa Medical University,

1-1 Daigaku, Uchinada, Ishikawa 920-0293, Japan

Full list of author information is available at the end of the article
}

identified as osteoblast lineage regulators (Stains and Civitelli 2003). Much progress has been made in understanding osteoblast differentiation, bone development, and the roles of bone morphogenetic protein (BMP), fibroblast growth factor (FGF), and the Wnt and JAK/ STAT signaling pathways (Long 2011; Long and Ornitz 2013; Raisz 2005). However, the underlying molecular mechanisms of osteoblast differentiation remain unclear. Sphingolipids are important components of mammalian cell membranes, and sphingomyelin (SM) is a major subspecies (Hannun and Obeid 2002; Merrill Jr. 2002; Spiegel and Milstien 2002). Sphingolipids are integral parts

(C) The Author(s). 2019 Open Access This article is distributed under the terms of the Creative Commons Attribution 4.0 International License (http://creativecommons.org/licenses/by/4.0/), which permits unrestricted use, distribution, and 
of various lipid membranes and serve as important second messengers for signal transduction pathways affecting cell growth and differentiation (Merrill $\mathrm{Jr}$ et al. 1997). Synthesis of SM from ceramide and phosphatidylcholine is mediated by the SM synthase (SMS) family members SMS1 and SMS2 (Huitema et al. 2004; Tafesse et al. 2006; Yamaoka et al. 2004). SMS1 is located in the Golgi apparatus and is responsible for bulk production of SM, whereas SMS2 is located in both the plasma membrane and Golgi apparatus (Jeckel et al. 1990; Villani et al. 2008; Yeang et al. 2008). Although enzymological differences between SMS1 and SMS2 are not clearly understood, SMS1 is known to be homeostatic, while SMS2 reacts to a diversity of stresses (Taniguchi and Okazaki 2014). Homozygous SMS1-mutant mice exhibit moderate neonatal lethality, reduced body weight, loss of fat-tissue mass, and growth deterioration, all of which suggest that they have metabolic abnormalities (Yano et al. 2011). In contrast, homozygous SMS2mutant mice do not exhibit overt physical abnormalities and show normal growth to adulthood. SMS2 deficiency inhibits induction of colitis-associated colon cancer (Ohnishi et al. 2017), ameliorates obesity induced by a high-fat diet ( $\mathrm{Li}$ et al. 2011), attenuates LPS-induced lung injury (Gowda et al. 2011), reduces expression of drug transporters in the brain (Zhang et al. 2011), and reduces atherosclerosis (Liu et al. 2009).

SM is hydrolyzed into ceramide and phosphatidylcholine by sphingomyelinases (Jung et al. 2000), which are classified into three categories: acidic, alkaline, and neutral (Nilsson and Duan 1999; Stoffel 1999). So far, no skeletal abnormalities in mice lacking SMPD1 or SMPD2 activity have been reported. SMPD3 encodes neutral sphingomyelinase 2, a membrane-bound enzyme whose expression is restricted to bone, cartilage, and brain (Khavandgar et al. 2011). Of the two SMPD3-deficient mouse models that have been established, one carries a chemically induced deletion of fro in the Smpd3 locus (fro/fro model) and the other was generated by gene targeting $\left(S M P D 3^{-/-}\right.$model) (Aubin et al. 2005; Guenet 1982; Stoffel et al. 2005). Both fro/fro and $S M P D 3^{-/-}$mice show severe bone and tooth mineralization defects as well as gross skeletal abnormalities. Fro/fro mice have been shown to have reduced ceramide levels in their skeletal tissues (Aubin et al. 2005).

Sphingolipids have been identified as growth-regulating molecules which regulate skeletal development and tissue homeostasis. The roles that SMS1 and SMS2 play in SM production and skeletal development are incompletely understood. SM is involved in matrix mineralization as well as signaling of extracellular stimuli. To clarify the role of SM in osteoblast biology, the genes essential to SM biosynthesis, SMS1 and SMS2, were knocked out in the osteoblast lineage. SMS1 and SMS2 are thought to play different roles in SM synthesis: SMS1 is a constitutive SM synthase in the
Golgi apparatus and SMS2 is a more regulated synthase on the plasma membrane. Not only constitutive SMS1 but also SMS2 genes are highly expressed in the osteoblast lineage. As a limitation of this study, it is still unclear whether SMS gene knock-out has an impact on the SM levels of osteoblasts in SMS-mutant mice. In this study we focused on how the SM-synthesis pathway affects bone morphogenesis and bone metabolism, and we determined the phenotype of

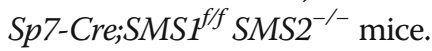

New conditional SMS2 knockout mice were generated by crossing Sp7 promotor-driven Cre-recombinase-expressing mice with mice homozygous for the floxed SMS1 gene. These $\mathrm{Sp} 7-\mathrm{Cre}$;SMS1 ${ }^{f f f} ; \mathrm{SMS2}^{-/-}$mice exhibit delayed ossification and osteoblast dysfunction, whereas SMS1/f; $S M S 2^{-/-}$mice show normal skeletal development similar to that of C57BL/6 mice. To clarify the role of SMS1 in osteogenesis, we also established an in vitro tamoxifen-inducible SMS1 inactivation system in osteoblasts using mice expressing a Cre-recombinase estrogen receptor (ERT2-Cre) fusion protein. Mutation of SMS1 was found, for the first time, to play an important role in osteoblast differentiation and mineralization stimulated by BMP2. Furthermore, mutation of SMS1 was shown to inhibit BMP2-induced Smad and MAPK signaling in osteoblasts.

\section{Materials and methods}

\section{Animal studies}

Animals were housed in a temperature-controlled room with a 12-h light/12-h dark cycle. Food and water were available ad libitum unless noted. All experimental protocols were approved by the Kanazawa Medical University Ethics Review Committee for Animal Experimentation. To generate $S M S 1^{f f f} ; S M S 2^{-/-}$mice, $S M S 1^{f / f}$ mice, in which SMS1 was flanked by loxP sequences, were crossed with $S M S 2^{-/-}$mice (Ohnishi et al. 2017). Sp7-green fluorescent protein/Cre mice were purchased from Jackson Laboratories (stock no. 006361; Bar Harbor, ME, USA). These two strains were crossed and maintained on a C57BL/6 J background. After generating triple heterozygous $\mathrm{Sp} 7-\mathrm{Cre}$; $S M S 1^{f f f} ; S M S 2^{+/-}$mice, they were bred with $S M S 1^{f f f}$; $S M S 2^{-/-}$mice to generate SMS1 conditional knockout mice (Sp7-Cre;SMS1 $\left.{ }^{f / f} ; S M S 2^{-/-}\right)$and Cre-null (SMS1 $1^{f / f}$; $S M S 2^{-/-}$) mice. To establish conditional whole-body knockout mice, $S M S f^{f f} ; S M S 2^{-/-}$mice were crossed with ERT2-Cre mice (stock no: 008463; Jackson Laboratories) to enable temporal control of floxed gene expression by tamoxifen induction. ERT2-Cre;SMS1 ${ }^{f / f} ; \mathrm{SMS}^{-/-}$mice were used for isolation and culture of primary calvarial osteoblasts. For genotyping, genomic DNA was isolated from mouse tails. Polymerase chain reactions (PCRs) were performed using a PCR Master Mix (Takara Bio, Inc., Shiga, Japan) with primer sequences for Cre transgene and floxed SMS1 genes relative to wild-type SMS1. 


\section{Skeletal preparation}

Embryonic day 15.5 mice (E15.5), 17.5 mice (E17.5) and newborn mice were eviscerated, fixed in $100 \%$ ethanol for 4 days and then transferred to acetone. After 3 days, specimens were rinsed with water and stained for 10 days in starting solution consisting of 1 volume of $0.1 \%$ Alizarin Red S (for bone) in 95\% ethanol, 1 volume of $0.3 \%$ Alcian blue 8GX (for cartilage) in 70\% ethanol, 1 volume of $100 \%$ acetic acid, and 17 volumes of ethanol. After rinsing with $100 \%$ ethanol, specimens were maintained in $1 \% \mathrm{KOH}$ at $37^{\circ} \mathrm{C}$ for $24 \mathrm{~h}$ and then maintained in $20 \%$ glycerol $/ 1 \%$ $\mathrm{KOH}$ at room temperature until the skeletons became clearly visible. For storage, specimens were transferred into 50, 80, and 100\% glycerol (Komori et al. 1997).

\section{Micro- and peripheral quantitative (pQ)-computed tomography (CT)}

We killed 15-week-old mice, and their femurs were fixed for 5 days in $100 \%$ ethanol before analyses by micro-CT (MCT-CB130F; Hitachi Medico, Tokyo, Japan) and pQCT (XCT Research SA+; Stratec Medizintechnik, Germany). We scanned femurs according to previously published procedures (Matsumoto et al. 2012).

\section{Histological and histomorphometric analyses}

The bones of 15 -week-old mice were fixed at $4{ }^{\circ} \mathrm{C}$ overnight in $4 \%$ paraformaldehyde and then decalcified with $10 \%$ EDTA for 10 days. Paraffin-embedded femurs were sectioned $(5-\mu \mathrm{m}$ thick) and stained with hematoxylin and eosin (H\&E). The overall width of the growth plate was measured from the metaphyseal margin of the terminal hypertrophic cell lacuna to the epiphyseal margin of the resting zone. Measurements were made as previously described (Marino et al. 2008). This measurement was performed in three areas of the growth plate and averaged. Non-decalcified, paraffin-embedded femur sections were evaluated by von Kossa staining using standard methods. Calcein double-labeling of femurs in mice was performed with calcein injections $\left(16 \mathrm{mg} \cdot \mathrm{kg}^{-1}\right.$ body weight) at 3-day intervals.

For histomorphometric analysis, femurs were excised, fixed with $75 \%$ ethanol, embedded in glycomethacrylate resin, and sectioned (3- $\mu \mathrm{m}$ thick). Sections were stained with toluidine blue and histomorphometrically analyzed under light microscopy with a micrometer, using a semiautomated image-analysis system (OsteoMeasure; OsteoMetrics, Atlanta, GA, USA). Parameters of the trabecular bone were measured in an area $1.28-2.09 \mathrm{~mm}^{2}$ in size from $1.125 \mathrm{~mm}$ above the growth plate at the distal metaphysis.

\section{Quantitative RT-PCR (qRT-PCR) analysis}

Total RNA and cDNA were prepared by ISOGEN ${ }^{\circ}$ (Wako, Osaka, Japan) and ReverTra Ace quantitative PCR Real-Time Master Mix (Toyobo, Osaka, Japan) according to the manufacturer's instructions. We performed qRT-PCR with a QuantStudio $12 \mathrm{~K}$ Flex RealTime PCR System (Thermo Fisher Scientific, Waltham, MA, USA) using TaqMan probes (Thermo Fisher Scientific) for collagen type I $\alpha 1$ (COL1A1; Mm00801666_g1), alkaline phosphatase (ALP; Mm00475834_m1), bone $\gamma$ carboxyglutamic acid (Gla) osteocalcin $(\mathrm{OCN})$ protein (OCN; Mm03413826_mH), secreted phosphoprotein 1 (OPN; Mm00436767_m1), integrin binding sialoprotein (BSP; Mm00492555_m1), receptor activator of nuclear factor $\kappa \mathrm{B}$ (RANK; Mm00435454_m1), tumor necrosis factor ligand superfamily, member 11 (RANKL; Mm00441908_m1), SMS1 (Mm00522643_m1), SMS2 (Mm00512327_m1), dentin matrix acidic phosphoprotein 1 (DMP1; Mm01208363_m1), phosphate-regulating endopeptidase homolog, X-linked (PHEX; Mm.2529), Osteoprotegerin (OPG; Mm00435454_m1) and TaqMan Master Mix (Thermo Fisher Scientific). Target gene expression was normalized to glyceraldehyde-3-phosphate dehydrogenase (GAPDH; Mm99999915_g1) expression.

\section{Isolation and culture of primary osteoblasts}

Calvarial bones from newborn ERT2-Cre;SMS1 fff; $S M S 2^{-/-}$and $S M S 1^{f / f} ; S M S 2^{-/-}$mice were collected by chipping with scissors. Pieces of bones were then digested twice with $0.1 \%$ collagenase type I (Wako Pure Chemical, Osaka, Japan) in phosphate-buffered saline at $37^{\circ} \mathrm{C}$ on a shaker for $15 \mathrm{~min}$. Subsequently, pieces of bones were digested three times in succession without pause with $0.1 \%$ collagenase for $20 \mathrm{~min}$, and osteoblastic populations were obtained from each solution to give fractions 3, 4, and 5 (Suto et al. 2012). Osteoblastic populations were centrifuged at $1500 \mathrm{rpm}$ for $5 \mathrm{~min}$, resuspended in $\alpha$-minimum essential medium ( $\alpha$-MEM) with $10 \%$ fetal bovine serum (FBS), $100 \mathrm{U} / \mathrm{mL}$ of penicillin, and $100 \mu \mathrm{g} / \mathrm{mL}$ of streptomycin. For cell-growth assays, osteoblasts were seeded on 24-well plates (IWAKI Cell Biology, Tokyo, Japan) at $2 \times 10^{4}$ cells/well. The next day, osteoblasts were treated with $1 \mu \mathrm{M}$ of 4hydroxytamoxifen (4-OHT) for $24 \mathrm{~h}$, treated with $5 \mathrm{ng}$ of FGF2 (R\&D Systems, Minneapolis, MN, USA), and allowed to proliferate for an additional 3 days. For differentiation assays, osteoblasts were seeded at a density of $2.5 \times 10^{4}$ cells $/ \mathrm{cm}^{2}$ on culture plates. Similar to the proliferation assay, the following day, osteoblasts were treated with 4-OHT and differentiation was induced by adding osteogenic medium ( $\alpha$-MEM containing 10\% FBS, $10 \mathrm{mM} \beta$-glycerophosphate, $50 \mu \mathrm{g} / \mathrm{mL}$ ascorbic acid, and $100 \mathrm{ng} / \mathrm{mL}$ BMP2). ALP staining was performed using a tartrate-resistant acid phosphatase (TRAP)/ALP staining kit (Wako Pure Chemical). ALP activity was measured in cell lysates using a LabAssay ${ }^{\mathrm{m}}$ ALP kit (Wako Pure Chemical). Gla-OCN synthesis was measured in culture medium using a mouse Gla-OCN 
competitive enzyme-linked immunosorbent assay kit (Takara Bio). To quantify terminal differentiation and mineralization of osteoblasts, cultured osteoblasts were fixed with phosphate-buffered formalin and then stained with $40 \mathrm{mM}$ Alizarin Red S (pH 4.2; Sigma-Aldrich) for $30 \mathrm{~min}$ at room temperature. After washing with distilled water, the plates were photographed. Alizarin Red S dye was extracted with $10 \%$ formic acid, and the absorbance at $415 \mathrm{~nm}$ was determined with a microplate reader.

\section{Western blot analysis}

Western blot analysis of cell extracts was performed as described previously (Ohnishi et al. 2017). Briefly, cells were washed with phosphate-buffered saline three times then lysed in RIPA buffer [ $50 \mathrm{mmol} / \mathrm{L}$ Tris- $\mathrm{HCl}(\mathrm{pH} 8.0)$, $150 \mathrm{mmol} / \mathrm{L} \mathrm{NaCl}, 0.5 \%$ (w/v) sodium deoxycholate, $0.1 \%(\mathrm{w} / \mathrm{v})$ sodium dodecyl sulfate, and 1.0\% (w/v) NP40 substitute]. After incubation on ice for $20 \mathrm{~min}$, debris was removed by centrifuging at $2000 \mathrm{~g}$ for $10 \mathrm{~min}$ at $4{ }^{\circ} \mathrm{C}$. Supernatant proteins $(20 \mu \mathrm{g})$ were electrophoresed with sodium dodecyl sulfate polyacrylamide gel and transferred to polyvinylidene fluoride membranes (Millipore, Billerica, MA, USA). Nonspecific binding was blocked by incubating membranes with Blocking One-P (Nacalai Tesque, Kyoto, Japan) for $20 \mathrm{~min}$ at room temperature. Then membranes were incubated overnight with primary antibodies, including anti-phospho-Smad1/ 5/8 (1:1000), anti-Smad1 (1:1000), anti-phospho-ERK1/2 (1:1000), and anti-ERK1/2 (1:1000), anti-phospho-p38 (1: 1000), anti-p38 (1:1000) [all from Cell Signaling Technology, Danvers, MA, USA], at $4{ }^{\circ} \mathrm{C}$ and then with secondary antibodies for $45 \mathrm{~min}$ at room temperature. Immunoreactive protein bands were visualized using an ECL-peroxidase detection system (Thermo Fisher Scientific) and LAS-4000 (Fujifilm, Tokyo, Japan).

\section{Statistical analysis}

The results are expressed as mean \pm standard deviation. Statistical comparisons between experimental groups were carried out using Student's $t$-test. A $P<0.05$ was considered statistically significant.

\section{Results}

\section{Osteoblast-specific deletion of SMS1 caused delayed ossification}

First, we generated double knockout (Sp7-Cre;SMS1 fff; $S M S 2^{-1-}$ ) mice by crossing $S p 7$ promoter-driven Crerecombinase-expressing mice with SMS1-floxed $S M S 2^{-1-}$ mice $\left(\mathrm{SMS1}^{f f f} ; \mathrm{SMS2}^{-/-}\right)$. There are no statistical differences between the skeletal phenotype and body weight of Sp7-Cre;SMS ${ }^{+/ f} ; \mathrm{SMS}^{+/-}$mice compared to C57BL/6 mice (data not shown). Alizarin Red S and Alcian blue staining of skeletal preparations was performed for E15.5 and newborn C57BL/6, SMSf ${ }^{f / f}$ SMS2 ${ }^{-/-}, \mathrm{Sp7-Cre}$ and Sp7-Cre;
$\mathrm{SMS1}^{f f f} ; \mathrm{SMS2}^{-/-}$mice. Newborn, E17.5 and E15.5 Sp7$\mathrm{Cre} ; \mathrm{SMS1}^{f / f} ; \mathrm{SMS2}^{-/-}$mice were smaller than littermate controls, but no significant patterning changes were observed in their bone or cartilage. In the early stages of ossification (E15.5), the calvaria and long limb bones of $\mathrm{Sp} 7-\mathrm{Cre}$;SMS1 ${ }^{\mathrm{fff}} ; \mathrm{SMS2}^{-/-}$mice showed poorer development, manifested by loosely mineralized bone structure and shorter long limb bones. At birth, mineralization of calvarial bone was delayed in $S p 7-C r e ; S M S 1^{f f f} ; S M S 2^{-/-}$ mice, but no skeletal defects were observed in $S M S 1^{f / f}$; $\mathrm{SMS2}^{-/-}$and Sp7-Cre mice. Moreover, Sp7-Cre;SMS1'ff; $S M S 2^{-/-}$newborns had less nasal bone development around the nasal capsule than did their littermate controls (Fig. 1a, b). No differences were observed in ossification between Sp7-Cre, SMSf ${ }^{f /} ; S M S 2^{-/-}$and C57BL/6 mice. These results are indicative of a bone-specific deficiency of SMS1 in Sp7-Cre;SMS1 fff; $\mathrm{SMS2}^{-/-}$mice. The effect of SMS1 and/or SMS2 deficiency on postnatal skeletal growth was evaluated in $\mathrm{Sp} 7-\mathrm{Cre} ; \mathrm{SMS1}^{f / f} ; \mathrm{SMS2}^{-/-}$mice relative to sex-matched littermate controls. As shown in Fig. 1c, d, the growth of Sp7-Cre;SMS1 ${ }^{f / f} ; S M S 2^{-/-}$mice was retarded.

\section{Sp7-Cre;SMS1 ${ }^{\mathrm{fff}} ; \mathrm{SMS2}^{-/-}$mice showed osteopenia}

Micro-CT analysis revealed microstructural changes in femurs of Sp7-Cre;SMSf ${ }^{f / f} ; S M S 2^{-/-}$mice, where bone forms via endochondral ossification. Micro-CT analysis of femurs in 15-week-old Sp7-Cre;SMS1 ${ }^{f / f} ; \mathrm{SMS2}^{-/-}$mice showed that trabecular bone parameters were markedly lower compared to C57BL/6, SMS1/f; $S M S 2^{-/-}, S p 7-C r e$; $\mathrm{SMS1}^{+/+} ; \mathrm{SMS2}^{+/-}$, and $\mathrm{Sp} 7-\mathrm{Cre} ; \mathrm{SMS1}^{+/+} ; \mathrm{SMS2}^{-/-}$mice. Compared with age-matched controls, the femurs of $\mathrm{Sp} 7$ $\mathrm{Cre} ; \mathrm{SMSP}^{f f f} ; \mathrm{SMS}^{-/-}$mice had reduced bone-volume fraction (77-80\% decrease) and decreased trabecular numbers (63-66\% decrease) accompanied by greater trabecular separation (Fig. 2a, b). In contrast, $S M S 1^{f f f} ; S M S 2^{-/-}, S p 7-$ $\mathrm{Cre} ; \mathrm{SMS1}^{+/+} ; \mathrm{SMS2}^{+/-}, \quad \mathrm{Sp} 7-\mathrm{Cre} ; \mathrm{SMS1}^{+/+} ; \mathrm{SMS2}^{-/-}$, and C57BL/6 mice showed no differences.

Next, we quantified cortical bone mineral density, cortical thickness, and the strength-strain index at the midshaft of femurs in 15-week-old mice by pQ-CT. These results revealed a reduced mineral density in both cortical and trabecular bones of Sp7-Cre;SMS1 $1^{f / f} ; S M S 2^{-/-}$ mice. Cortical thickness was also reduced by $30 \%$ in femurs of Sp7-Cre;SMS1 $1^{f f f}$;SMS2 ${ }^{-/-}$mice versus that of controls. Bone strength was established with respect to torsion and found to be significantly lower at the distal femoral shaft of Sp7-Cre;SMSf ${ }^{f / f} ; \mathrm{SMS2}^{-/-}$mice than in controls (Fig. 2c, d). Collectively, these quantitative micro- and pQ-CT results agree with qualitative images, suggesting reduced trabecular and cortical bone mineralization in $\mathrm{Sp} 7-\mathrm{Cre} ; \mathrm{SMS1}{ }^{f / f} ; \mathrm{SMS2}^{-/-}$mice. These results imply that skeletal abnormalities in Sp7-Cre; $S M S 1^{f / f} ; S M S 2^{-/-}$mice persist as these mice age. 
A

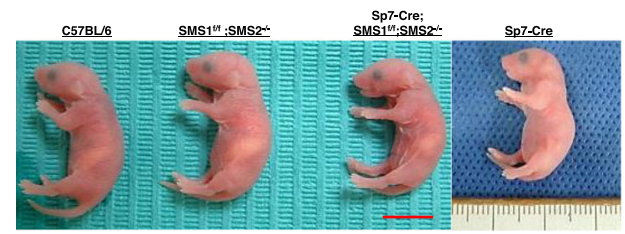

C

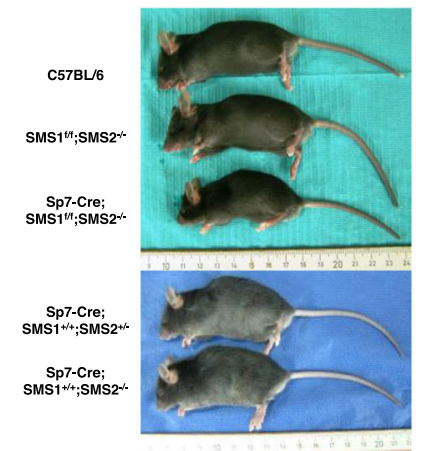

B

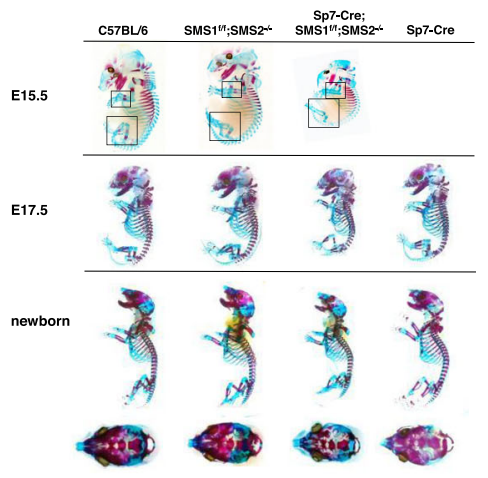

D

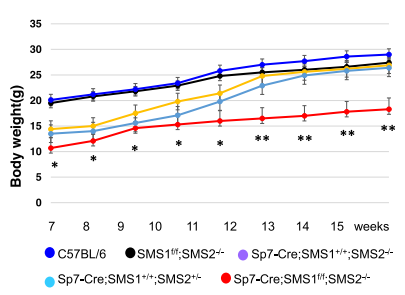

Fig. 1 Skeletal changes and growth retardation in Sp7-Cre;SMS1 ${ }^{f / f} ; S M S 2^{-/-}$mice. a Gross appearance of C57BL/6, SMS1 ${ }^{f / f} ; S M S 2^{-/-}$, Sp7-

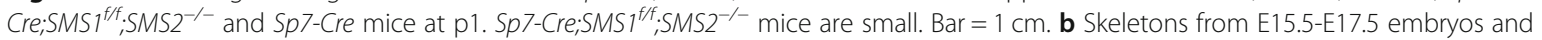
newborns (p1) of C57BL/6, SMS1 ${ }^{f / f} ; S_{M S 2}{ }^{-1-}, S p 7-C r e ; S M S 1^{f / f} ; S M S 2^{-1-}$ and Sp7-Cre mice. Embryos and newborns were stained with Alizarin red and Alcian blue. Note the delayed mineralization of the limbs in the Sp7-Cre;SMS ${ }^{f / f} ;$ SMS2 ${ }^{-1-}$ E15.5 embryo compared with that of C57BL/6 and $\mathrm{SMS}^{\mathrm{ff}}{ }_{\text {; }} \mathrm{SMS2}^{-1-}$ mice (black square). Dorsal views of skulls of newborns showed that calvarial bone was hypomineralized in Sp7-

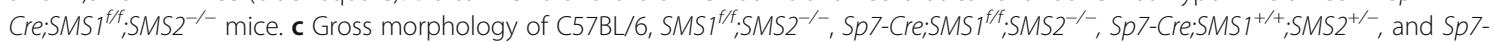

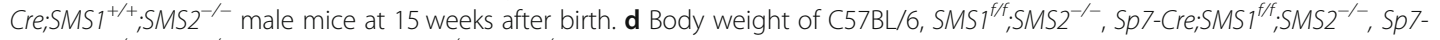

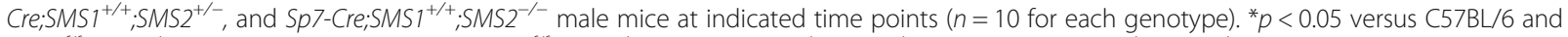

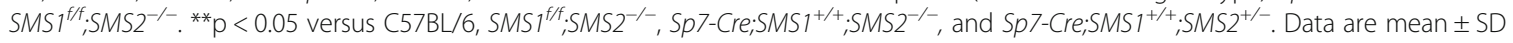

\section{Mutation of SMS1 reduced bone mineralization rates}

$\mathrm{H} \&$ E-stained sections of distal femurs revealed that trabecular bone mass near the growth plate of Sp7-Cre; $S M S 1^{f / f} ; \mathrm{SMS}^{-/-}$mice was reduced than those of $\mathrm{C} 57 \mathrm{BL} /$ 6, SMS1 ${ }^{f / f} ; \mathrm{SMS}^{-/-}$and Sp7-Cre;SMS1 ${ }^{+/+} ; \mathrm{SMS}^{+/-}$mice. The epiphyseal growth plate and cartilaginous tissues of Sp7-Cre;SMS1 ${ }^{+/+} ; S M S 2^{+/-}$and Sp7-Cre;SMS1 flf,$S M S 2^{-/-}$ mice were thinner than those of control mice, however, there was no significant difference (Fig. 3a, b).

To further clarify the effect of SMS1 deficiency on reduced bone mass and BMD, we compared in vivo mineral apposition in Sp7-Cre;SMS1 $1^{f / f} ; S M S 2^{-/-}$and control mice via calcein double-labeling. A smaller distance between the calcein-labeled mineralization front at the midshaft of femurs was observed in Sp7-Cre;SMS1 f/f; $S M S 2^{-1-}$ mice versus controls, suggesting less new bone formation in Sp7-Cre;SMS1 ${ }^{f / f} ; S M S 2^{-/-}$mice. In undecalcified Von Kossa staining, the osteoid was stained red and mineralized bone substance appeared black. Greater osteoid formation was seen in Sp7-Cre;SMS1 ${ }^{f / f} ; \mathrm{SMS}^{-/-}$ mice compared to control mice (Fig. 3c).
Histomorphometric analysis showed that the mineral apposition rate (MAR), bone formation rate (BFR/BS), and mineralized surface (MS/BS) of Sp7-Cre;SMS1 f/f; $S M S 2^{-/-}$mice were $25-40 \%, 40-55 \%$ and $30-40 \%$ lower, respectively, than those of controls. It also revealed that osteoblast-related parameters (osteoid volume/bone volume (OV/BV), osteoid surfaces (OS/BS), and osteoblast surfaces (ob. S/BS) were significantly increased in Sp7Cre;SMS1 ${ }^{f / f}$;SMS2 $2^{-/-}$mice compared to controls. In contrast, osteoclast-related parameters eroded surfaces (ES/ BS), osteoclast numbers (N.Oc/B.Pm), and osteoclast surfaces (Oc.S/BS) were not affected (Fig. 3d). Combined with the micro- and $\mathrm{pQ}-\mathrm{CT}$ data above, the results suggest that a lower bone mineralization rate and an increase in the number of osteoids contributed, at least in part, to the lower bone mass found in Sp7-Cre;SMS1 flf; $S M S 2^{-/-}$mice. To gain further insight into the potential mechanisms underlying defective bone mineralization in Sp7-Cre;SMS1 $1^{f / f}$;SMS2 $2^{-/-}$mice, we compared gene expression in femur bones of C57BL/6, SMS1 fff; $\mathrm{SMS2}^{-/-}, \mathrm{Sp7-Cre}$; $\mathrm{SMS1}^{+/+} ; \mathrm{SMS}^{+/-}, \mathrm{Sp} 7-\mathrm{Cre}$;SMS1 ${ }^{+/+} ; \mathrm{SMS}^{-/-}$, and Sp7-Cre; 

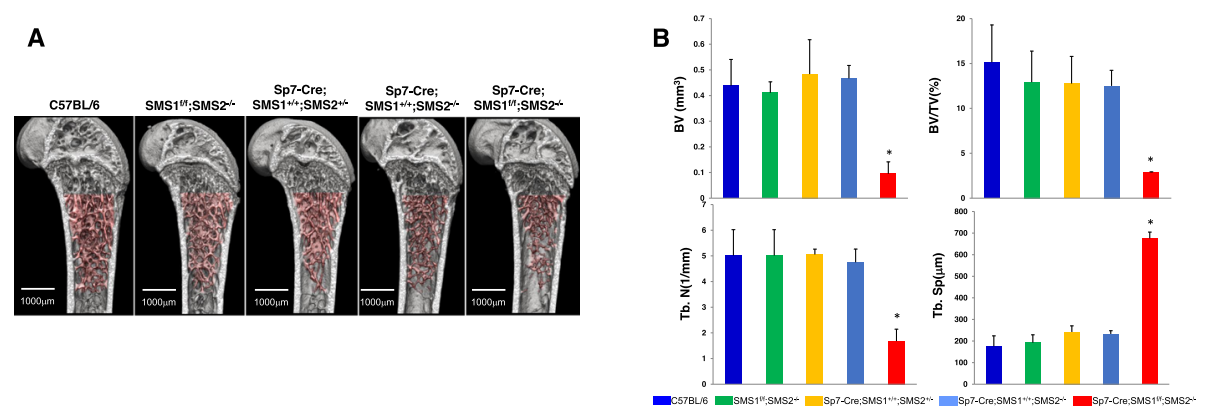

C

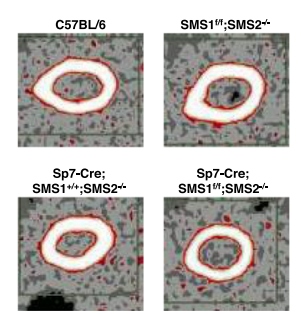

D

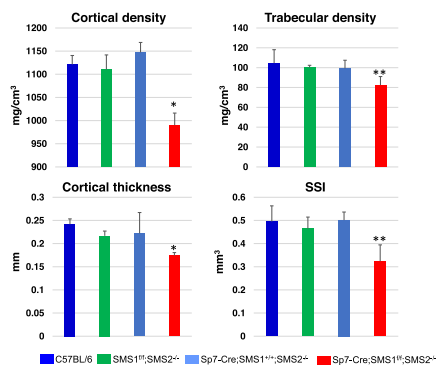

Fig. 2 Note the osteoporotic phenotype of the $S p 7-C r e ; S M S 1^{f / f} ; S M S 2^{-/-}$mice. a Representative micro-CT images of the distal femoral shafts of 15-

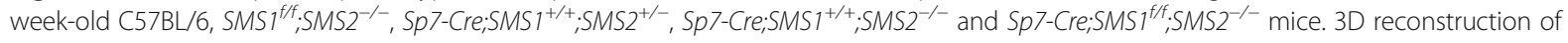
trabecular bone. b Micro-CT analysis of femurs from 15-week-old mice. Abbreviations: BV, bone volume; BV/TV, bone volume/total volume; Tb N,

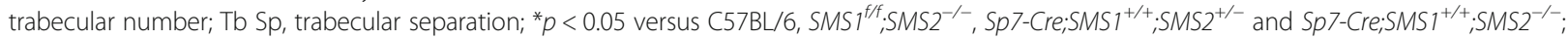
mean $\pm S D, n=10$ for each genotype. $\mathbf{c}$ Isolated femurs of 15-week-old mice were scanned using $\mathrm{pQCT}$. Sections were made at the midshaft. $\mathbf{d}$ Quantification of cortical density, trabecular density, cortical thickness and strength strain index (SSI). ${ }^{*} p<0.005$ versus C57BL/6, SMS1 ${ }^{f / f}$;SMS2 ${ }^{-1-}$,

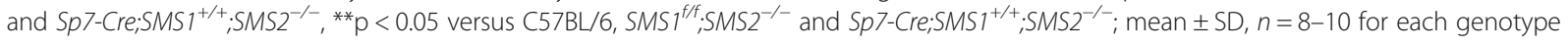

$S M S f^{f f} ; S_{S M S 2^{-/-}}$mice at postnatal day 5 using qRT-PCR. The results showed that COL1A1, ALP, OCN, OPN, and BSP mRNA were downregulated significantly, while a mild downregulation of DMP1 and PHEX expression was observed (Fig. 3e). Next, we measured the gene expression of RANK, RANKL, and OPG, and we computed the RANKL/ OPG ratio. Interestingly, RANK, RANKL, OPG, and the RANKL/OPG ratio were mildly downregulated but not statistically significantly in Sp7-Cre;SMS1f/f;SMS2-/- mice compared to control mice (Fig. 3f).

\section{Inactivation of SMS1-impaired BMP2-induced osteoblast differentiation}

To gain further insight into how SMS1 affects the activity of primary neonatal osteoblasts, we investigated whether loss of SMS1 activity affects the in vitro mineralization capacity of cultured osteoblasts. Because bone formation of $\mathrm{Sp} 7$-Cre;SMS1 ${ }^{f f} ; \mathrm{SMS}^{-/-}$mice was poor, sufficient osteoblast numbers could not be obtained from $\mathrm{Sp7-Cre;}$ $S M S 1^{f f f} ; \mathrm{SMS2}^{-/-}$calvarial bone. We also established a system to induce ablation of SMS1 in osteoblasts from ERT2Cre;SMS1 ${ }^{\text {fff }} ; \mathrm{SMS}^{-/-}$mice in vitro. ERT2-Cre mice were crossed to enable tracking and visualization of Cremediated inactivation of SMS1. By crossing SMSt ff; $S M S 2^{-/-}$mice with those mice, tamoxifen-inducible SMS1-deficient $S M S 2^{-/-}$mice (ERT2-Cre;SMS1 ${ }^{f f f} ; S_{S S 2^{-/-}}$) were created that were able to provide sufficient neonatal calvarial osteoblasts; ERT2-Cre negative control calvarial osteoblasts were prepared from $S M S 1^{f f f} ; S M S 2^{-/-}$mice. An analysis by qRT-PCR showed that SMS1 expression was decreased in osteoblasts treated with 4-OHT (Fig. 4b). The above system was also used to determine whether induced inactivation of SMS1 affected osteoblast proliferation and differentiation. First, we examined the cell growth activity of osteoblasts as shown in the scheme in Fig. 4a. After a 4day treatment with 4-OHT, the cell number was slightly increased. Addition of FGF2 increased the cell number further in both $S M S 1^{f f f} ; S M S 2^{-/-}$and ERT2-Cre;SMS1 flf; $S M S 2^{-1-}$ osteoblasts (Fig. 4c). These results indicated that the ability to stimulate the growth of osteoblasts with FGF2 was not affected by deletion of SMS1. Next, we examined the differentiation of osteoblasts (Fig. 5a). ALP activity and Gla-OCN synthesis, both of which are early osteoblast differentiation markers, were then analyzed (Fig. 5b, c). ALP activity and Gla-OCN synthesis were significantly increased 4 days after stimulation with BMP2 in SMS1 fff $\mathrm{SMS2}^{-/-}$and ERT2-Cre;SMS1 fff; $\mathrm{SMS2}^{-/-}$osteoblasts without 4-OHT treatment. In contrast, there was no BMP2-stimulated increase in either ALP activity or Gla-OCN synthesis in 4-OHT-treated ERT2-Cre;SMS1 ${ }^{\mathrm{ff} ;}$; $\mathrm{SMS2}^{-/}$osteoblasts. An analysis by qRT-PCR showed that addition of BMP2 significantly 


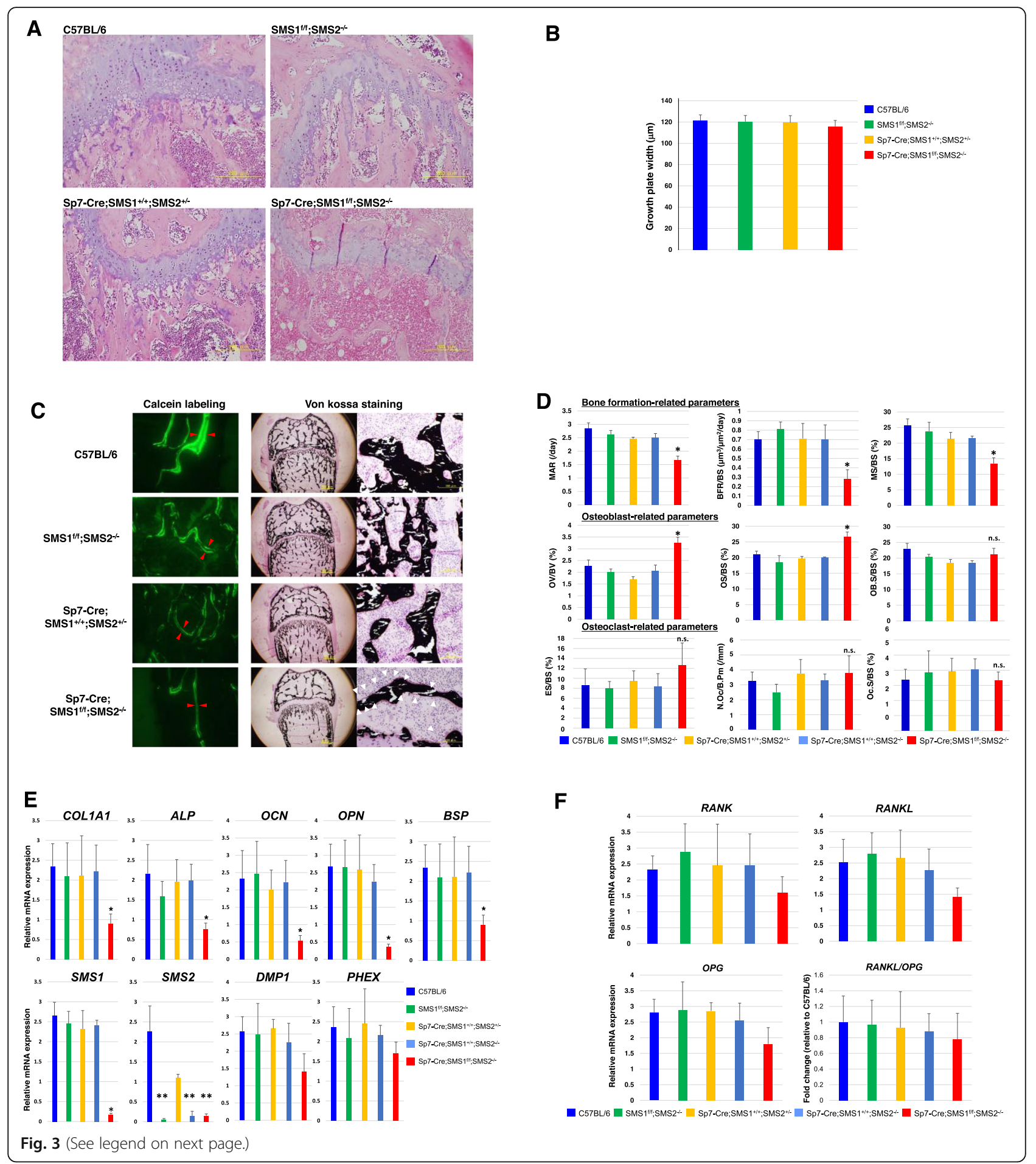


(See figure on previous page.)

Fig. 3 Decreased bone formation in adult Sp7-Cre;SMS1/f/SMS2 ${ }^{-/-}$mice. a Hematoxylin-eosin staining of the distal femurs of 15-week-old C57BL/

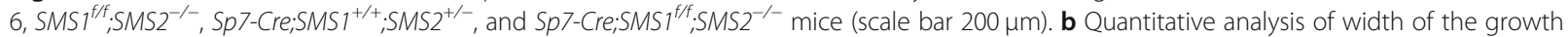

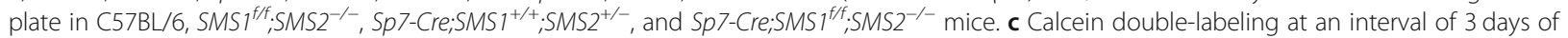

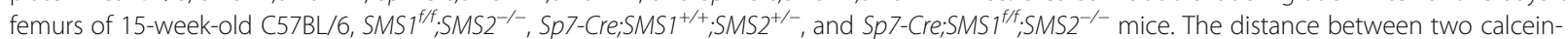
labeled mineralization fronts is indicated by red arrow heads at the midshaft of femurs (left). Von Kossa staining of the distal femurs of 15-week-

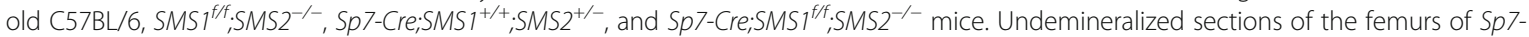
$\mathrm{Cre} ; \mathrm{SMSI}^{\mathrm{fff}} ; \mathrm{SMS}^{-1-}$ mice show that there is little trabecular interconnection (middle), that bone mass is almost normal, and that there is extensive accumulation of osteoid (right). Mineralized bone is stained black, unmineralized osteoid is stained in red. Osteoid is indicated by white arrow

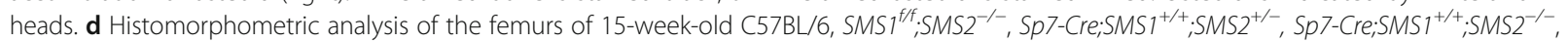

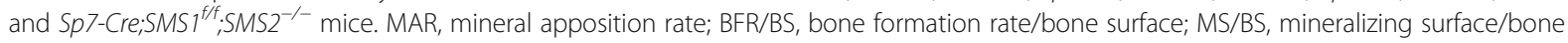
surface; OV/OB, osteoid volume/bone volume; OS/BS, osteoid surface/bone surface; Ob.S/BS, Osteoblast surface/bone surface; ES/BS, eroded surface/bone surface; N.OC/B.Pm, Osteoclast numbers/osteoclast perimeter; Oc.S/BS, osteoclast surface/bone surface; ${ }^{*}, p<0.05$ versus C57BL/6,

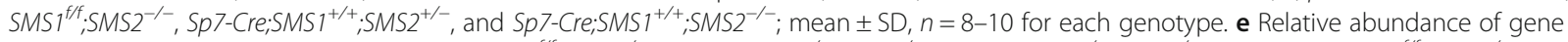

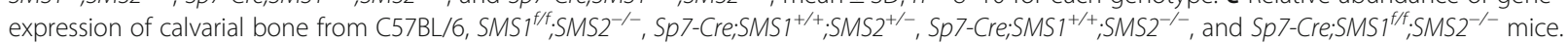
Significant downregulation of COL1A1, ALP, OCN, OPN, and BSP expression was observed in Sp7-Cre;SMS1f/f;SMS2-/- mice. f Relative abundance of RANK, RANKL, and OPG expression and the RANKL/OPG ratio for each mouse. Quantitative analysis of RT-PCR using RNA from p5 calvarial

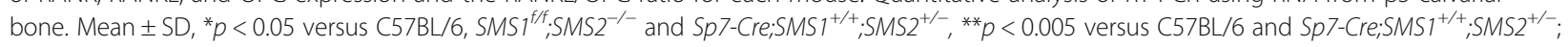
$n=10$ for each genotype

increased COL1A1, BSP, and OPN mRNA expression levels in $S M S 1^{f / f} ; S M S 2^{-/-}$and ERT2-Cre;SMS1 ${ }^{f / f} ; S M S 2^{-/-}$ osteoblasts without 4-OHT. However, 4-OHT-treated ERT2-Cre;SMS1 $1^{f / f}$;SMS2 ${ }^{-/-}$osteoblasts showed reduced COL1A1, BSP, and OPN mRNA expression after BMP2 treatment (Fig. 5d). In mineralization assays, quantification of Alizarin Red S staining was used for terminal differentiation of osteoblasts. Osteoblasts were treated in the same manner as for ALP assays, except that osteoblasts were analyzed 14 days after stimulation with BMP2. After stimulating with BMP2, SMS1 $1^{f / f} ; S M S 2^{-/-}$and ERT2-Cre;SMS1 flf ;SMS2 ${ }^{-/-}$osteoblasts without 4-OHT became significantly mineralized. In contrast, ERT2Cre;SMS1 ${ }^{f / f}$;SMS2 $2^{-/-}$osteoblasts treated with 4-OHT were less mineralized than those without 4-OHT treatment (Fig. 5e). These data suggest that inactivation of SMS1 inhibits the BMP2-induced differentiation of osteoblasts.

\section{A}

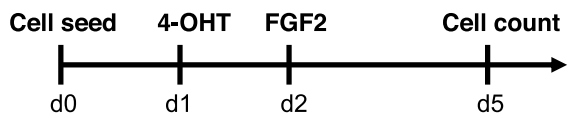

B

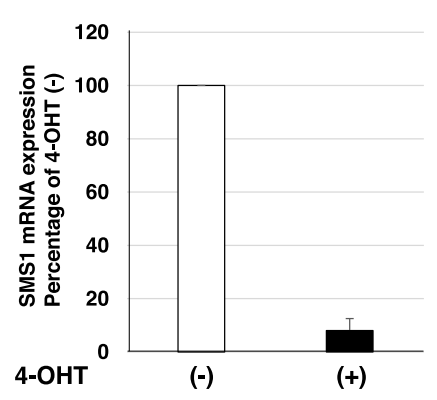

C

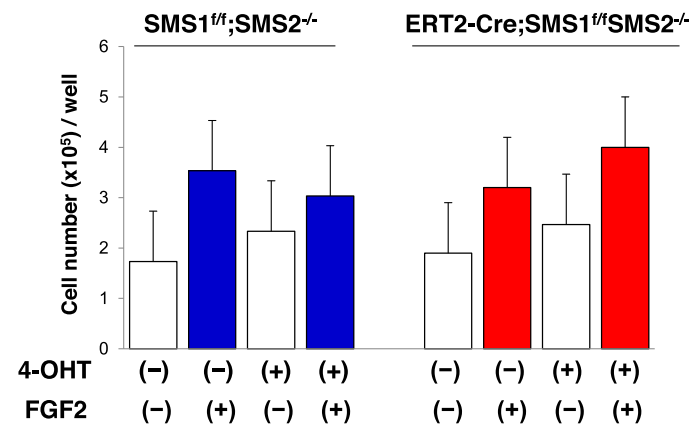

Fig. 4 Effects of SMS1 knock-out on osteoblast cell growth. a Schematic outline of the experimental design. Calvarial osteoblast cells were seeded on 48-well plates (5000 cells/well) with 10\% FBS containing a-MEM. b Reduction of expression of SMS1 in osteoblasts treated with $1 \mu$ M 4-

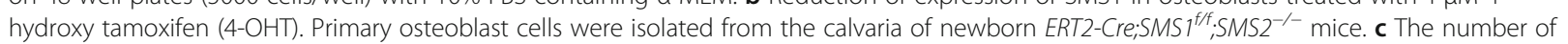
cells was counted 4 days after treatment with 4-OHT. Inactivation of SMS1 resulted in no growth inhibition of calvarial osteoblasts. Treatment with 4-OHT does not affect the cell growth of primary SMS1/f; $S M S 2^{-/-}$calvarial osteoblasts. Osteoblasts were prepared from newborn SMS ${ }^{f / f} ; S M S 2^{-/-}$ and ERT2-Cre;SMS1 ${ }^{\mathrm{fff}} ; \mathrm{SMS2}^{-/-}$mice. Values are expressed as the mean $\pm \mathrm{SD}, n=8$ for each genotype 

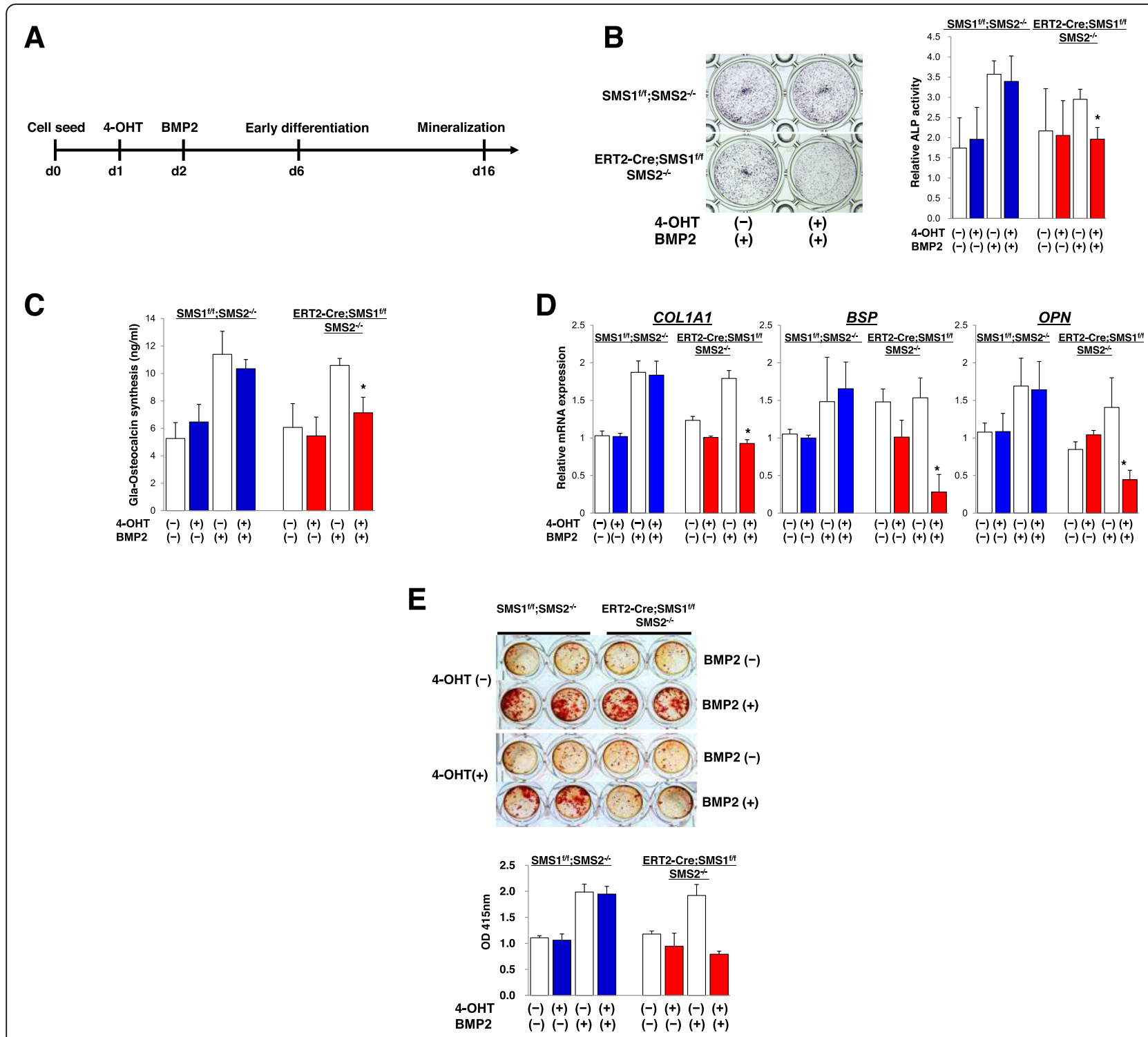

Fig. 5 Effects of SMS1 knock-out on osteoblast differentiation. a Schematic outline of the experimental design. b Representative pictures of ALPstaining of primary calvarial osteoblast cultures in the absence or presence of $1 \mu \mathrm{M} 4-\mathrm{OHT}$ at day 6 after BMP2 stimulation (left). Quantification of the staining is shown (right). Inactivation of SMS1 resulted in reduced ALP activity of calvarial osteoblasts. ${ }^{*} p<0.005$ versuS SMS1 ${ }^{f / f}$; $S M S 2^{-1-}$ (4$\mathrm{OHT}+, \mathrm{BMP} 2+)$ and ERT2-Cre;SMS1 ${ }^{\mathrm{ff}} ; \mathrm{SMS2}^{-1-}(4-\mathrm{OHT}-, \mathrm{BMP} 2+)$; Values are expressed as the mean $\pm \mathrm{SD}, n=8$. c Gla-Osteocalcin contents of primary calvarial osteoblast cultures in the absence and presence of $4-\mathrm{OHT}$ at day 6 after BMP2 stimulation. ${ }^{*} p<0.005$ versus SMS1/f/FSS2 ${ }^{-1-}(4-\mathrm{OHT}+$,

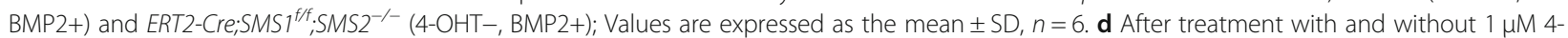
OHT for 1 day, $S M S 1^{f / f} ; S M S 2^{-1-}$ and ERT2-Cre;SMS1 ${ }^{\mathrm{f} / f} ; \mathrm{SMS2}^{-1-}$ mice calvarial osteoblasts were cultured with and without $100 \mathrm{ng} / \mathrm{ml}$ BMP2 in a-MEM with $10 \%$ FBS containing $50 \mathrm{\mu g} / \mathrm{ml}$ ascorbic acid, $10 \mathrm{mM}$ B-glycerophosphate for 4 days. Col1a1, BSP and OPN mRNA levels were normalized to GAPDH mRNA. Treatment with 4-OHT does not affect the differentiation of primary SMS1/f;SMS2 ${ }^{-1-}$ calvarial osteoblasts. ${ }^{*} p<0.005$ versus

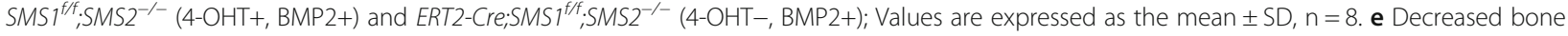
nodule formation in the 4-OHT-induced SMS1 inactivation. Representative images of nodule mineralization stained with Alizarin red. $S M S 1^{f / f} ; S M S 2^{-/-}$and ERT2-Cre;SMS1 ${ }^{f / f} ; S_{M S 2^{-/-}}$mice calvarial osteoblasts were treated with and without $1 \mu M$ 4-OHT for 1 day, and cultured with and without $100 \mathrm{ng} / \mathrm{ml} \mathrm{BMP2}$. On day 17, the cells were stained with Alizarin red (upper). Quantification of the staining is shown (lower). ${ }^{*} p<$

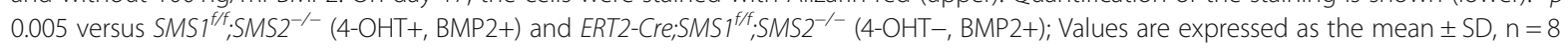


Inactivation of SMS1 inhibits BMP2-induced phosphorylation of Smad1/5/8 and p38 in primary osteoblasts

To gain further insight into the potential mechanism by which inactivation of SMS1 inhibits BMP2-induced osteoblast differentiation, we next examined the effect of SMS1 deficiency on BMP2 signaling pathways [i.e., Smad and mitogen-activated protein kinase (MAPK)] in primary osteoblasts. BMP2 stimulation rapidly induced phosphorylation of intracellular Smad1/5/8, Erk1/2, and p38, key downstream molecules of BMP2 signal transduction. When ERT2-Cre;SMS1 ${ }^{f / f}$;SMS2 $2^{-/-}$calvarial osteoblasts were treated with 4-OHT, BMP2induced Smad1/5/8 phosphorylation was significantly inhibited. Next, we examined the effect of inactivation of SMS1 on MAPK signaling. We showed that BMP2induced p38 phosphorylation was mildly inhibited but Erk1/2 phosphorylation was unaffected by inactivation of SMS1 (Fig. 6). These results showed impaired BMP2 signaling in SMS1-deficient osteoblasts, suggesting that SMS1 functions to regulate osteoblast activity in cooperation with BMP2 signaling.

\section{Discussion}

SM is generated by SMS1 and SMS2. Function of both SMS1 and SMS2 in different organs were studied by SMS1 and SMS2 knockout mice. However, it remains unknown whether SMS1 and/or SMS2 has a direct effect in bone formation. The goals of the current study are to characterize the skeletal phenotype of mice that lack SMS1 and/or SMS2 and to investigate the local roles SMS1 and SMS2 play in osteoblasts. In this study, we generated conditional knockout mice by crossing Sp7 promoter-driven Cre-recombinase-expressing mice and mice homozygous for the floxed SMS1 gene. SP7 (osterix) is required for an important step in the differentiation of pre-osteoblasts into fully functional osteoblasts (Nakashima and de Crombrugghe 2003). Using the SP7-Cre drive to conditionally inactivate SMS1 in pre-osteoblasts and block SMS1 function in these cells, we found a low-bone-mass phenotype with decreased trabecular bone and inhibited osteoblast differentiation in this study. Although SP7-Cre mice suffer delayed growth of cortical bone and low body weight in the early stage of development, these effects have largely disappeared by 12 weeks of age when the mice are skeletally mature (Davey et al. 2012). Wang et al. reported that there were severe intramembranous bone-formation defects in SP7-Cre mice during the early postnatal stage but that mineralization gradually improved during development and had become almost completely normal by 3 weeks of age (Wang et al. 2015). Additionally, we used control SP7-Cre mice in our study when appropriate.

It is clear that SMS1 is important for whole-body health, especially considering that SMS1-deficient mice exhibit moderate neonatal lethality (Yano et al. 2011). To our knowledge, SMS1 conditional knockout in osteoblasts has not been investigated in Sp7 promoter-driven

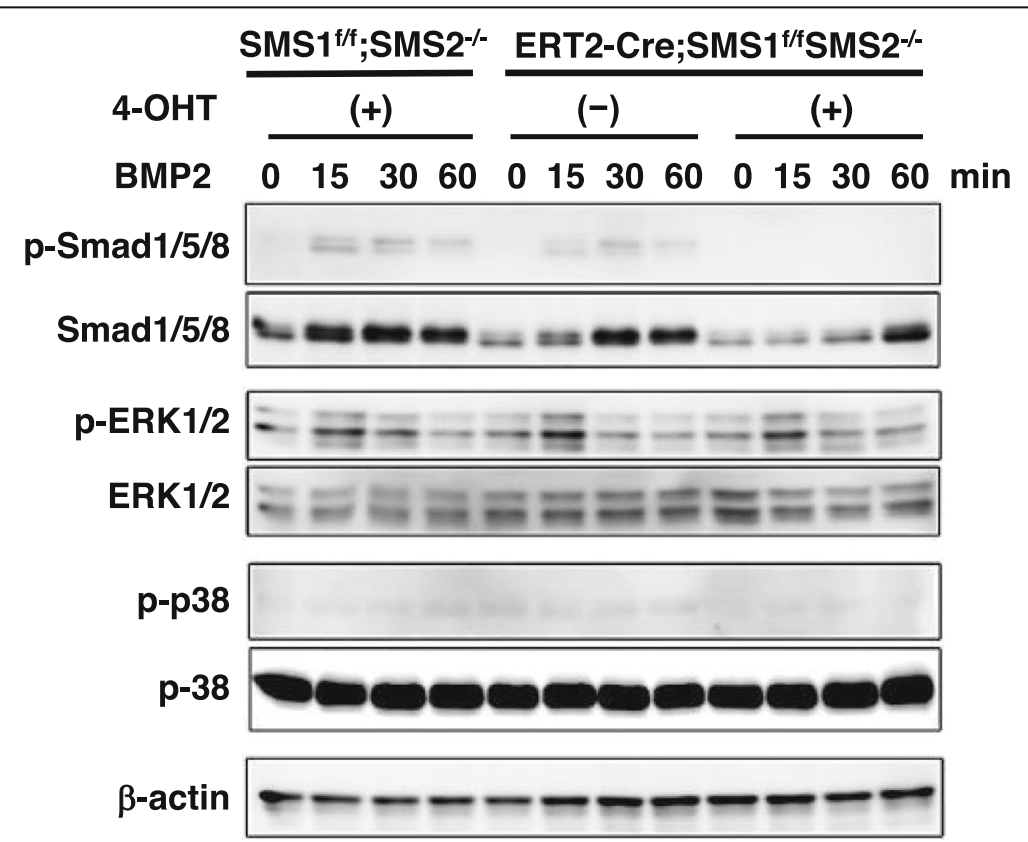

Fig. 6 Inactivation of SMS1 inhibited BMP2-induced Smad1/5/8 and p38 phosphorylation. The cellular phosphorylation levels of Smad1/5/8, ERK1/

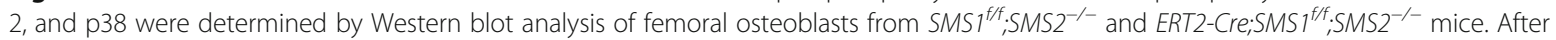
treatment with and without $1 \mu \mathrm{M}$ 4-OHT for 1 day, calvarial osteoblasts were serum starved for $12 \mathrm{~h}$ before BMP2 (50 ng/ml) stimulation for the indicated times, and then immunoblotted with antibodies specific to phospho-Smad1/5/8, phospho-ERK1/2, and phospho-p38 
Cre mice. In this study, we have demonstrated for the first time that SMS1 conditional knockout (Sp7-Cre; $\left.S M S 1^{f / f} ; S M S 2^{-/-}\right)$causes bone development defects, while SMS2 knockout $\left(S M S 1^{f f f} ; S M S 2^{-/-}\right)$does not. After birth, there is a difference in body weight between Sp7Cre-positive mice and Sp7-Cre-negative mice. However, SP7-Cre-positive littermate controls have delayed growth characterized by lower body weight until 10 weeks of age, but this delay in growth is overcome by the time adulthood is reached at 12 weeks. We found that the volume of cortical and trabecular bone in our Sp7controlled conditional SMS1 knockout mice was decreased, indicating that their overall weight loss and growth retardation may primarily result from bone abnormalities in adulthood. On the other hand, SMS2 knockout mice are characterized neither by reduced body weight nor a reduced volume of cortical and trabecular bone. These results imply that SMS2 does not affect bone development. However, it is unknown whether SMS1 has a direct functional role in bone formation. In the present study, we have addressed this question by demonstrating the effect of bone-specific deletion of SMS1 on postnatal skeletal development and thus establishing the function of SMS1 to regulate the development of osteoblasts. We found that the skeletal phenotype of E15.5, E17.5, newborn, and 15-week-old Sp7-controlled conditional SMS1 knockout mice exhibit reduced mineralization of calvaria and long limb bones in the early stages of ossification and reduced trabecular number, trabecular bone volume, and cortical bone thickness in vivo. On the other hand, $\mathrm{SMS}^{-/-}$and SP7Cre-positive littermate controls showed no reduction of trabecular number, trabecular bone volume, or cortical bone thickness in vivo. These results imply that Sp7controlled conditional SMS1 knockout mice have decreased bone mass, which results in bone defects during pre- and postnatal growth.

Cortical bone thickness was reduced in affected $S p 7$ $\mathrm{Cre} ; \mathrm{SMS1}^{\mathrm{ff}} ; \mathrm{SMS}^{-/-}$mice compared to control mice. Although there was no increased osteoid in adult $S M S 1^{f / f}$; $\mathrm{SMS}^{-/-}, \mathrm{Sp} 7-\mathrm{Cre} ; \mathrm{SMS1}^{+/+} ; \mathrm{SMS2}^{+/-}$mice, the amount of osteoid was mildly increased in adult Sp7-Cre;SMS1 fff; $S M S 2^{-/-}$mice. However, Von Kossa stained sections

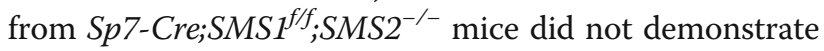
characteristic features of hypophosphatemic rickets including increased width of the unmineralized epiphyseal growth plate and increased trabecular bone volume in the proximal metaphysis. The growth-plate regions demonstrated normal cartilage calcification in the hypertrophic zone and at the epiphyseal end of the growth plate in Sp7-Cre;SMS1 ${ }^{f / f} ; S M S 2^{-/-}$mice. In Von Kossastained sections, we consistently observed a tendency for

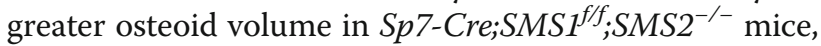
and histomorphometric analysis revealed that this was statistically significant. These results imply that the osteoblast differentiation process was affected in $S p 7$ $\mathrm{Cre} ; \mathrm{SMS1}^{\mathrm{fff}} ; \mathrm{SMS2}^{-/-}$mice.

In bone, RANKL is expressed in osteoblasts and their precursors, while RANK is present on the cell-surface membranes of osteoclasts and their progenitors (Nagy and Penninger 2015). It is therefore a marker of bone resorption. In this study, in femoral bone of Sp7-Cre;SMSiff; $S M S 2^{-/}$mice, mature ALP (osteoblast marker) expression level was significantly downregulated, while RANKL (osteoblast functional marker) and RANK (osteoclast marker) expression levels were slightly downregulated. RANK and RANKL were slightly less compared to control mice, showing that the resorption process in $\mathrm{Sp} 7-\mathrm{Cre}$; $S M S 1^{f f f} ; S_{S S 2^{-/-}}$mice was limited or retarded. OPG, the marker of bone protection against erosion, showed a mild downregulation compared to control mice. This trend of OPG is correlated with the decrease of RANK and RANKL in $\mathrm{Sp} 7-\mathrm{Cre} ; \mathrm{SMSI}^{f f} ; \mathrm{SMS2}^{-/-}$mice. The RANKL/OPG ratio is considered a comprehensive indicator of prevailing activity of bone remodeling (Canciani et al. 2017). The RANKL/OPG ratio is negative not only in $\mathrm{Sp} 7-\mathrm{Cre}$;SMS1 f/f; $S M S 2^{-/-}$but in control mice as well. It might be that bone apposition is prevalent compared to resorption. Furthermore, ALP activity in cultured Sp7-Cre;SMS1ff; $\mathrm{SMS2}^{-/-}$ osteoblasts was significantly decreased, while TRAP activity was unaltered. TRAP staining of femur sections revealed that the number of TRAP-positive multinucleated osteoclasts was unchanged in $\mathrm{Sp} 7-\mathrm{Cre} ; \mathrm{SMSI}^{\mathrm{ff}} ; \mathrm{SMS2}^{-/-}$mice; this finding was confirmed by histomorphometric analysis. Osteoclast-related parameters, the percentage of bone surface covered by mature osteoclasts (Oc.S/BS), and the number of mature osteoclasts (N.Oc/B.Pm) were comparable to controls. To determine the role of SMS1 in osteoclast development, bone-marrow-derived monocyte/ macrophage precursor cells (BMMs) isolated from C57BL/ 6, $S M S 1^{f f f} ; \mathrm{SMS}^{-/-}$, and Sp7-Cre;SMS1 ${ }^{f f f} ; \mathrm{SMS}^{-/-}$mice were treated with mM-CSF and mRANKL in vitro. Osteoclast differentiation was not affected in Sp7-Cre;SMS1 f/f; $S M S 2^{-/}$BMMs as indicated by TRAP staining and activity (data not shown). These data suggest that decreased trabecular bone volume in $\mathrm{Sp} 7-\mathrm{Cre} ; \mathrm{SMS1}^{f / f} ; \mathrm{SMS}^{-/-}$mice is not due to increased bone resorption. The histomorphometric analysis of the present study revealed a significantly increased number of osteoids in trabecular bone, with a slightly increased number of osteoclasts, in Sp7-Cre; $S M S 1^{f / f} ; S M S 2^{-/-}$mice, but not in $S M S{ }^{f / f} ; S M S 2^{-/-}$mice or SP7-Cre-positive littermate control mice. These results imply that arrested osteoblast development contributes, at least in part, to reduced bone formation observed in Sp7controlled conditional SMS1 knockout mice.

Primary neonatal osteoblast culture is a wellestablished cell-culture system to analyze in vitro osteoblast differentiation. The observation that $\mathrm{Sp} 7$-controlled 
conditional SMS1 knock-out caused significantly compromised osteoblast mineralization in vivo raised the question of whether it would affect osteoblast differentiation and mineralization in vitro. Wang et al. showed that both early and terminal differentiation of primary calvarial osteoblasts isolated from neonatal SP7-Cre mice were comparable to those of control cells (Wang et al. 2015). In the present study, to investigate the mechanisms underlying bone defects in $S p 7-\mathrm{Cre}$;SMS1 $1^{f / f} ; \mathrm{SMS2}^{-/-}$mice, we focused on the response to BMP2-induced osteoblast differentiation and mineralization in primary osteoblasts. We found that after BMP2 stimulation 4-OHT-treated SMS1-inactivated osteoblasts were significantly decreased in ALP activity, BSP and OPN expression, and Gla-OCN synthesis. These results suggest that impaired bone formation is not caused by the inhibition of osteoblast proliferation but rather by suppression of early and late stages of osteoblast differentiation.

Next, we found suppression of phospho-Smad1/5/8 and phospho-p38, but not phospho-ERK1/2, in 4-OHT-treated SMS1-inactivated osteoblasts after BMP2 stimulation. Smad family members play major roles in transforming signaling in the growth-factor- $\beta$ superfamily (Macias et al. 2015; Retting et al. 2009). BMP2-induced phosphorylation of Smad1/5/8 cooperates with Runx2 (Leong et al. 2009; Schroeder et al. 2005), and Runx2 is an essential osteoblastrelated gene regulator that promotes expression of ALP (Lemonnier et al. 2004). Therefore, activation of Smad1/5/8 is required for osteoblast differentiation. MAPKs, such as p38 and ERK1/2, were also activated by BMP2 in our study and have been documented to participate in the differentiation-inducing effects of BMP2 in MC3T3 and primary osteoblasts (Egwuagu 2009). Moreover, MAPK signaling influences the development of osteoblast differentiation (Xiao et al. 2002a, b), and FGF signaling is known to regulate osteoblast proliferation via MAPK pathways (Ornitz and Itoh 2015). P38 signaling has been shown to play a major role in endochondral bone development (Stanton et al. 2004).

Recently, it has been reported that BMP2 regulates SMPD3 expression in osteoblasts via p38 mitogenactivated protein kinase and its downstream transcription factors. However, the major role of BMP2 signaling involving SMAD1/5/8 may not have a direct effect on the transcriptional regulation of SMPD3 (Manickam et al. 2019). In the present study, in which ERT2-Cre;SMS1 ${ }^{\mathrm{f} / \mathrm{f}} ; \mathrm{SMS}^{-/-}$ osteoblasts were treated with 4-OHT, BMP2-induced phosphorylation of both Smad1/5/8 and p38 was inhibited. Previous reports have shown that the amount of sphingomyelin species was decreased, whereas the amounts of ceramide species and glycosphingolipid were increased in SMS1-KO islets, suggesting that accumulated ceramide species were alternatively metabolized into glycosphingolipid species. However, there was no change of expression of SMPD3 mRNA in SMS1-KO islets (Yano et al. 2011). Considering these, SMS1 ablation may not be a direct regulator of SMPD3 expression, although sphingolipid metabolism may regulate BMP2-induced osteoblast maturation. In the present study, 4-OHT treatment affected differentiation in response to BMP2 in ERT2-Cre;SMS1 ${ }^{\mathrm{ff} f} ; \mathrm{SMS2^{-/- }}$ primary osteoblasts. These findings demonstrate an in vivo interaction between SMS1 and BMP2 in bone and suggest that SMS1 regulates osteoblast development in neonatal and postnatal bone formation through cooperation with BMP2 signaling. Our data identifying Smad1/5/8 and p38 as regulators of SMS1 expression imply that SMS1 may act as a downstream effector of Smad1/5/8 and p38. However, further studies are required to understand how various transcription factors cross talk to the BMPs in SMS1-regulated bone formation.

\section{Conclusion}

The results of our current study suggest that knocking out SMS1 in the SP7-expressing osteoprecursors impairs bone development and retards growth. Both of these effects are associated with the regulation of SMS1 in the development of osteoblasts. Further studies are needed to understand how various transcription factors crosstalk to regulate SMS1 expression in bone formation.

\section{Abbreviations}

4-OHT: 4-hydroxytamoxifen; ALP: Alkaline phosphatase; BMMs: Bone-marrowderived monocyte/macrophage precursor cells; BMP: bone morphogenetic protein; BSP: Integrin binding sialoprotein; COLIA1: Collagen type I a1; CreERT2: Cre-recombinase estrogen receptor; DMP1: dentin matrix acidic phosphoprotein 1; FGF: fibroblast growth factor; GAPDH: Glyceraldehyde-3phosphate dehydrogenase; OCN: Osteocalcin; OPG: Osteoprotegerin; OPN: Secreted phosphoprotein 1; PHEX: phosphate regulating endopeptidase homolog, X-linked; RANK: Receptor activator of nuclear factor KB; RANKL: Tumor necrosis factor ligand superfamily, member 11; Runx2: runt-related transcription factor-2; SM: sphingomyelin; SMS: sphingomyelin synthase

\section{Acknowledgements}

We are grateful to Martin Peters. He critically revised the article for important intellectual contents.

\section{Authors' contributions}

$\mathrm{GM}, \mathrm{CH}, \mathrm{KW}$, and MT contributed to study design, laboratory work, data analysis, and statistical analysis and drafted the article. GM, KW and TO conceived the study, contributed to its design and coordination. GM and TO secured funding. All authors read and approved the final article.

\section{Funding}

This work was supported, in part, by grants from the Strategic Research Foundation Grant-Aided Project for Private Universities (Grant S1201004) of the Japan Ministry of Education, Culture, Sports, Science, and Technology (MEXT) and by a Grant-in-Aid for Scientific Research (C) 16 K11710 from MEXT.

\section{Availability of data and materials}

All data produced or analyzed during this study are included in this published article.

\section{Ethics approval and consent to participate}

All experimental protocols were approved by the Kanazawa Medical University Ethics Review Committee for Animal Experimentation. All animals were maintained in a specific pathogen-free (SPF) grade animal room. 
Animals were housed in a temperature-controlled room with a 12-h light/12h dark cycle. Food and water were available ad libitum unless noted.

\section{Consent for publication}

All authors give the consent for the publish of this study.

\section{Competing interests}

The authors declare that they have no competing interests.

\section{Author details}

'Department of Oral and Maxillofacial Surgery, Kanazawa Medical University, 1-1 Daigaku, Uchinada, Ishikawa 920-0293, Japan. ²Department of Medicine, Division of General and Digestive Surgery, Kanazawa Medical University, Ishikawa, Japan. ${ }^{3}$ Department of Bone and Joint Disease, National Center for Geriatrics and Gerontology, Aichi, Japan. ${ }^{4}$ Department of Life Science, Medical Research Institute, Kanazawa Medical University, Ishikawa, Japan. ${ }^{5}$ Research Institute for Bioresources and Biotechnology, Ishikawa Prefectural University, Ishikawa, Japan.

Received: 25 July 2019 Accepted: 25 November 2019

Published online: 17 December 2019

\section{References}

Aubin I, et al. A deletion in the gene encoding sphingomyelin phosphodiesterase 3 (Smpd3) results in osteogenesis and dentinogenesis imperfecta in the mouse. Nat Genet. 2005;37:803-5.

Canciani $\mathrm{E}$, et al. RNA isolation from alveolar bone and gene expression analysis of RANK, RANKL and OPG: a new tool to monitor bone remodeling and healing in different bone substitutes used for prosthetic rehabilitation. Arch Oral Biol. 2017;80:56-61.

Davey RA, et al. Decreased body weight in young Osterix-Cre transgenic mice results in delayed cortical bone expansion and accrual. Transgenic Res. 2012;21:885-93.

Ducy P, Schinke T, Karsenty G. The osteoblast: a sophisticated fibroblast under central surveillance. Science. 2000;289:1501-4.

Egwuagu CE. STAT3 in CD4+ T helper cell differentiation and inflammatory diseases. Cytokine. 2009:47:149-56.

Gowda S, et al. Sphingomyelin synthase 2 (SMS2) deficiency attenuates LPSinduced lung injury. Am J Physiol lung cell Mol. Physiol. 2011;300:L430-40.

Guenet JL. Fragilitas ossium (fro): an autosomal recessive mutation in the mouse. Prog Clin Biol Res. 1982;94:265-7.

Hannun YA, Obeid LM. The Ceramide-centric universe of lipid-mediated cell regulation: stress encounters of the lipid kind. J Biol Chem. 2002;277:25847-50.

Huitema K, van den Dikkenberg J, Brouwers JF, Holthuis JC. Identification of a family of animal sphingomyelin synthases. EMBO J. 2004;23:33-44.

Jeckel D, Karrenbauer A, Birk R, Schmidt RR, Wieland F. Sphingomyelin is synthesized in the cis Golgi. FEBS Lett. 1990;261:155-7.

Jung SY, et al. Identification of multiple forms of membrane-associated neutral sphingomyelinase in bovine brain. J Neurochem. 2000;75:1004-14.

Khavandgar Z, et al. A cell-autonomous requirement for neutral sphingomyelinase 2 in bone mineralization. J Cell Biol. 2011;194:277-89.

Komori T, et al. Targeted disruption of Cbfa1 results in a complete lack of bone formation owing to maturational arrest of osteoblasts. Cell. 1997;89:755-64.

Lemonnier J, Ghayor C, Guicheux J, Caverzasio J. Protein kinase Cindependent activation of protein kinase $\mathrm{D}$ is involved in BMP-2-induced activation of stress mitogen-activated protein kinases JNK and p38 and osteoblastic cell differentiation. J Biol Chem. 2004;279:259-64.

Leong WF, Zhou T, Lim GL, Li B. Protein palmitoylation regulates osteoblast differentiation through BMP-induced osterix expression. PLoS One. 2009;4:e4135.

$L i Z$, et al. Reducing plasma membrane sphingomyelin increases insulin sensitivity. Mol Cell Biol. 2011;31:4205-18.

Liu J, et al. Macrophage sphingomyelin synthase 2 deficiency decreases atherosclerosis in mice. Circ Res. 2009;105:295-303.

Long F. Building strong bones: molecular regulation of the osteoblast lineage. Nat Rev Mol Cell Biol. 2011;13:27-38.

Long F, Ornitz DM. Development of the endochondral skeleton. Cold Spring Harb Perspect Biol. 2013;5:a008334.

Macias MJ, Martin-Malpartida P, Massagué J. Structural determinants of Smad function in TGF- $\beta$ signaling. Trends Biochem Sci. 2015;40:296-308.

Manickam G, Moffatt P, Murshed M. Role of SMPD3 during bone fracture healing and regulation of its expression. Mol Cell Biol. 2019;39:e00370-18.
Marino R, et al. Catch-up growth after hypothyroidism is caused by delayed growth plate senescence. Endocrinology. 2008;149:1820-8.

Matsumoto $G$, et al. Bone regeneration by polyhedral microcrystals from silkworm virus. Sci Rep. 2012;2:935.

Merrill AH Jr, et al. Sphingolipids--the enigmatic lipid class: biochemistry, physiology, and pathophysiology. Toxicol Appl Pharmacol. 1997;142:208-25.

Merrill AH Jr. De novo sphingolipid biosynthesis: a necessary, but dangerous pathway. J Biol Chem. 2002;277:25843-6.

Nagy V, Penninger JM. The RANKL-RANK story. Gerontology. 2015;61:534-42.

Nakashima K, de Crombrugghe B. Transcriptional mechanisms in osteoblast differentiation and bone formation. Trends Genet. 2003;19:458-66.

Nilsson A, Duan RD. Alkaline sphingomyelinases and ceramidases of the gastrointestinal tract. Chem Phys Lipids. 1999;102:97-105.

Ohnishi T, et al. Sphingomyelin synthase 2 deficiency inhibits the induction of murine colitis-associated colon cancer. FASEB J. 2017;31:3816-30.

Ornitz DM, Itoh N. The fibroblast growth factor signaling pathway. Wiley Interdiscip Rev Dev Biol. 2015;4:215-66.

Raisz LG. Pathogenesis of osteoporosis: concepts, conflicts, and prospects. J Clin Invest. 2005;115:3318-25.

Retting KN, Song B, Yoon BS, Lyons KM. BMP canonical Smad signaling through Smad1 and Smad5 is required for endochondral bone formation. Development. 2009:136:1093-104.

Schroeder TM, Jensen ED, Westendorf JJ. Runx2: a master organizer of gene transcription in developing and maturing osteoblasts. Birth Defects Res C: Embryo Today. 2005;75:213-25.

Spiegel S, Milstien S. Sphingosine 1-phosphate, a key cell signaling molecule. J Biol Chem. 2002;277:25851-4.

Stains JP, Civitelli R. Genomic approaches to identifying transcriptional regulators of osteoblast differentiation. Genome Biol. 2003;4:222-4.

Stanton LA, Sabari S, Sampaio AV, Underhill TM, Beier F. p38 MAP kinase signalling is required for hypertrophic chondrocyte differentiation. Biochem J. 2004;378:53-62.

Stoffel W. Functional analysis of acid and neutral sphingomyelinases in vitro and in vivo. Chem Phys Lipids. 1999;102:107-21.

Stoffel W, Jenke B, Blöck B, Zumbansen M, Koebke J. Neutral sphingomyelinase 2 (smpd3) in the control of postnatal growth and development. Proc Natl Acad Sci U S A. 2005;102:4554-9.

Suto $\mathrm{K}$, et al. Repeated freeze-thaw cycles reduce the survival rate of osteocytes in bone-tendon constructs without affecting the mechanical properties of tendons. Cell Tissue Bank. 2012;13:71-80.

Tafesse FG, Ternes P, Holthuis JC. The multigenic sphingomyelin synthase family. J Biol Chem. 2006;281:29421-5.

Taniguchi M, Okazaki T. The role of sphingomyelin and sphingomyelin synthases in cell death, proliferation and migration-from cell and animal models to human disorders. Biochim Biophys Acta. 2014;1841:692-703.

Villani $\mathrm{M}$, et al. Sphingomyelin synthases regulate production of diacylglycerol at the Golgi. Biochem J. 2008;414:31-41.

Wang L, Mishina Y, Liu F. Osterix-Cre transgene causes craniofacial bone development defect. Calcif Tissue Int. 2015;96:129-37.

Xiao G, et al. Bone morphogenetic proteins, extracellular matrix, and mitogenactivated protein kinase signaling pathways are required for osteoblastspecific gene expression and differentiation in MC3T3-E1 cells. J Bone Miner Res. 2002a;17:101-10.

Xiao G, Jiang D, Gopalakrishnan R, Franceschi RT. Fibroblast growth factor 2 induction of the osteocalcin gene requires MAPK activity and phosphonylation of the osteoblast transcription factor, Cbfa1/Runx2. J Biol Chem. 2002b;277:36181-7.

Yamaoka S, Miyaji M, Kitano T, Umehara H, Okazaki T. Expression cloning of a human CDNA restoring sphingomyelin synthesis and cell growth in sphingomyelin synthase-defective lymphoid cells. J Biol Chem. 2004;279:18688-93.

Yano $M$, et al. Mitochondrial dysfunction and increased reactive oxygen species impair insulin secretion in sphingomyelin synthase 1-null mice. J Biol Chem. 2011;286:3992-4002.

Yeang C, et al. The domain responsible for sphingomyelin synthase (SMS) activity. Biochim Biophys Acta. 2008;1781:610-7.

Zhang Y, Dong J, Zhu X, Wang W, Yang Q. The effect of sphingomyelin synthase 2 (SMS2) deficiency on the expression of drug transporters in mouse brain. Biochem Pharmacol. 2011;82:287-94.

\section{Publisher's Note}

Springer Nature remains neutral with regard to jurisdictional claims in published maps and institutional affiliations. 